\title{
Advances in Research on Bladder Cancer Targeting Peptides: a Review
}

\author{
Bin Zheng $\mathbb{D}^{1,2} \cdot$ Pu Zhang $\mathbb{D}^{2} \cdot$ Heng Wang ${ }^{2} \cdot$ Jinxue Wang ${ }^{3} \cdot$ Zheng Hong Liu ${ }^{1} \cdot$ DaHong Zhang $\mathbb{D}^{1}$
}

Accepted: 27 June 2021 / Published online: 1 September 2021

(c) The Author(s) 2021

\begin{abstract}
Bladder cancer (Bca) is the second most common malignant tumor of the genitourinary system in Chinese male population with high potential of recurrence and progression. The overall prognosis has not been improved significantly for the past 30 years due to the lack of early theranostic technique. Currently the early theranostic technique for bladder cancer is mainly through the intravesical approach, but the clinical outcomes are poor due to the limited tumor-targeting efficiency. Therefore, the targeting peptides for bladder cancer provide possibility to advance intravesical theranostic technique. However, no systematic review has covered the wide use of the targeting peptides for intravesical theranostic techniques in bladder cancer. Herein, a summary of original researches introduces all aspects of the targeting peptides for bladder cancer, including the peptide screening, the targeting mechanism and its preclinical application.
\end{abstract}

Keywords Bladder tumor $\cdot$ Peptides targeting $\cdot$ Diagnosis $\cdot$ Treatment $\cdot$ Research progress

\section{Introduction}

Bladder cancer (Bca) is the second most common malignant tumor of the genitourinary system in Chinese male population with high potential of recurrence and progression up to $21-31 \%$ and $0.8-3.8 \%$, respectively [1]. Currently a confirmed diagnosis of $\mathrm{BCa}$ mainly relies on cystoscopy or transurethral resection of bladder tumor combined with pathological examination [2]. On some occasion where nonmuslce $\mathrm{BCa}$ (NMIBC) is of highly aggressive type, longterm intravesical is strongly recommended [2]. Therefore, at least $80 \%$ of $\mathrm{BCa}$ population benefit from the intravesical theranostic technique. However, both the surveillance of transurethral endoscope and intravesical therapy are lack of tumor specificity. Hence, carcinoma in situ or atypical hyperplasia can be ignored on the vision of white light

These authors contributed equally: Bin Zheng, Pu Zhang

DaHong Zhang

zhangdahong88@126.com

1 Zhejiang Chinese Medical University, 310053 HangZhou, China

2 Zhejiang Provincial People's Hospital, Hangzhou Medical College, 310014 Hangzhou, China

3 Handan Central hospital, 056001 Handan, China cystoscopy, and drugs intravesically administrated can not be concentrated in the malignant lesion. The key to improve the efficacy of intravesical theranostic technique is to equip itself with targeting agent.

The currently used targeting agents can be mainly categorized into nucleic acid aptamers, antibodies, and peptides. Nucleic acid aptamers can be screened out to target many types of elements, including inorganic and organic small molecules, biological macromolecules, and even cells. But it is sometimes challenging to obtain nucleic acid aptamers with high specificity and high affinity for individual targets [3]. Due to the electronegative nature of the nucleic acid aptamers, the binding affinity for electronegative target wanes [4]. Its binding affinity also requires certain ionic strength, $\mathrm{pH}$, and so forth [4]. The antibody exhibits good performance in good specificity, but its value is discounted by its obvious toxicity to normal tissues [5], poor penetration into tumor tissues [6,7], and high cost of prodcution. The targeting peptide usually composes of no more than 100 monomeric amino acids. The cost of production is lower compared to that of antibodies. The high binding affinity can be maintained in harsh conditions. Besides, the lower molecular weight of the targeting peptide determined its facilitated elimination from nonspecific targeting tissues at the concentration of nanomolar. From the perspective of stability, specificity and economical cost, the targeting peptide is more potential to be used for modify the 
intravesical theranostic technique. However, no systematic review has covered the wide use of the targeting peptides for intravesical theranostic techniques in Bca. Herein, all aspects of the targeting peptides for Bca is introduced, including the peptide screening, the targeting mechanism, and its preclinical application.

\section{Screening of Peptide}

\section{Phage-Displayed Peptide Libraries}

One of the most widely used peptide screening technology is phage-displayed technology, featured by its high efficiency and low cost. Smith first proposed this technology in 1985 [8]. The mechanism behind this can be simplified as a combination of the genetic engineering technology and the peptide library with phage. In 1990, Scott and Smith successfuly introduced the fusion protein gIII containing random sequence peptides on the phage surface [9] to complete a phage surface display peptide library. Specifically, it is necessary to insert a chemically synthesized random oligonucleotide sequence into the gene encoding the phage coat protein to express various random short peptides Table 1. Then, the phages were panned for bladder tumor cells, and phages that specifically bind to bladder tumors were screened out. Amplify the DNA insert of the phage clone by polymerase chain reaction (Polymerase Chain Reaction, PCR), and sequence the DNA insert to convert it into the corresponding peptide sequence. The phage-displayed technology was used to screened out many types of $\mathrm{BCa}$ targeting peptides, including $\mathrm{P} 4$ peptide, Bld-1 peptide, CLT1 peptide, and NYZL1 peptide [10-13].

\section{One-Bead One-Compound Combinatorial Peptide Library Technology}

The one-bead, one-peptide synthesis method is another peptide screening technology. One resin bead serves as a carrier, loading with a peptide of one sequence, and the One bead and one peptide (a resin bead contains a peptide sequence) are synthesized using resin as a carrier and according to the mixing-equalization method Table 2. After each step is connected to the resin, it is insoluble in the solvent, causing impurities. Excess reagents are easy to remove. After screening and sequencing of the obtained peptide library, the peptide structure can be obtained $[14,15]$. The principle of the onebead one-peptide library made by the mixing-equalization method is to first connect the protected amino acids to the resin, such as ten kinds of amino acids, after being mixed and deprotected, they are divided into ten groups, and each group has ten kinds connected to the resin. Then, ten protected amino acids are used to couple with each group to obtain $10 \times 10$ dipeptides (connected with resin), and the resulting dipeptides are mixed and divided into ten groups evenly. After repeating the above steps, 10X10X10 peptides can be obtained. Then use the established peptide library to develop tumor-specific targeting peptides. Simultaneously screen millions of beads (each bead is a unique peptide sequence) to identify peptides bound to tumor cell surface molecules. We can use an enzyme-linked colorimetric method similar to western blot or observe the cells attached to the beads' surface to screen for "positive beads" with specific ligands. The PLZ4 peptide was obtained by co-cultivating the one-bead-one-peptide library with $\mathrm{BCa}$ cells after two-round screening, and has high affinity to BCa cells as proved in T24, 5637, and TCCSUP cell lines [16].

\section{Derived Peptide}

The targeting peptide can also be a form of a natural ligand or a peptide derivative, such as $\mathrm{pH}$ low insertion peptide (pHLIP peptide). pHLIP is a water-soluble polypeptide derived from helix $\mathrm{C}$ of bacteriorhodopsin, and can insert into the cell membrane to form a stable transmembrane $\alpha$-helix at the acidic tumor microenvironment [17]. Similarly, NT-4 peptide is a four-branched peptide containing human neurotensin sequence with short retention period in the circulation [18]. For prolonged circulation retention, several types of neurotensin analogs have been developed [19-21], and much more concentrated accumulation in BCa tissues was verified [18].

Fibronectin Attachment Protein (FAP) is a member of the fibronectin-binding glycoprotein family, and plays an essential role in the process of Bacillus Calmette-Guérin adhesion to BCa. The functional, active region of FAP is located in sequence 269-280, and the binding sequence is RWFV [22].

The purified HIV nuclear trans activating factor (TAT), a classical type of the cell-penetrating peptide (CPP), can be spontaneously internalized into the cell without a transfection agent in the cell medium [23]. The arginine-rich fragment in the 48-60 sequence of TaT protein is a crucial component to break through the membrane barrier independent of the receptor-mediated and the energy-dependent endocytosis pathway [24]. Arginine polymers composing of eleven arginines (R11) mimics the active part of the TAT peptide, and targets $\mathrm{BCa}$ specifically rather than other tumors [25].

\section{Peptide Targeting Mechanism}

\section{Tumor Microenvironmental Response}

Tumor cells are mainly metabolized by aerobic glycolysis to meet a strong energy demand, leading to the accumulation 


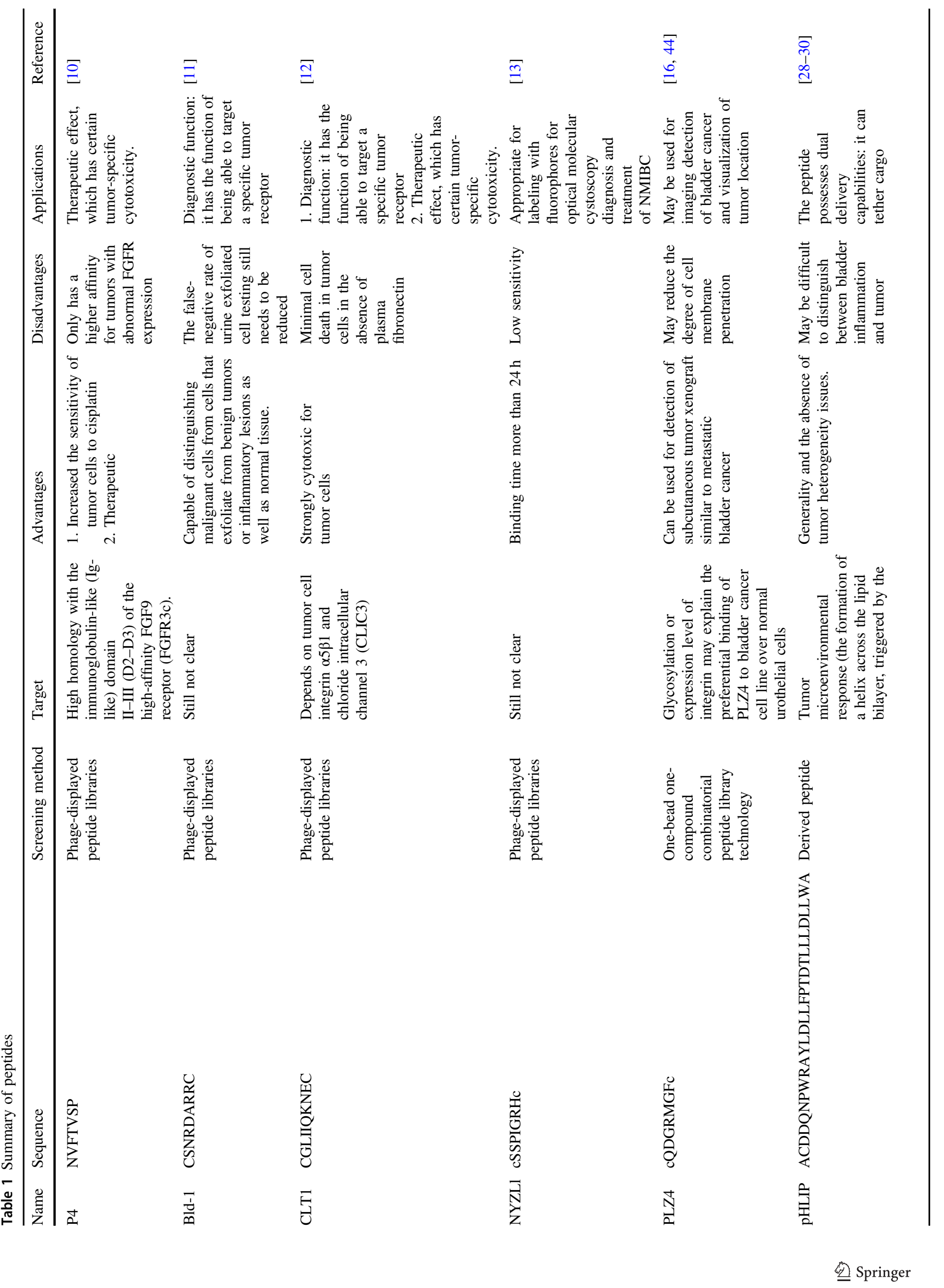


of the metabolic product lactic acid and the decreased $\mathrm{pH}$ value of the tumor tissue area being 0.3-0.7 $\mathrm{U}$ lower than normal tissues [26]. The pHLIP peptide is sensitive to targeting in an acidic environment and can detect changes in the body from $0.2-0.3 \mathrm{pH}$ units [27, 28]. The acidic environment caused by the high metabolism of bladder tumors meets the conditions for specific targeting of PHLIP. For $\mathrm{pHLIP}$, the reduced $\mathrm{pH}$ triggered the protonation of Asp residue and the increased hydrophobicity of the peptide chain. Under this condition, pHLIP can form its spiral structure easily across the bilayer membrane [29]. Studies on clinical specimens have confirmed the specific targeting of PHLIP to the acidic environment of bladder tumors [30].

\section{Receptor and Ligand Interaction}

The cyclic peptide PLZ4 (amino acid sequence cQDGRMGFc) can binds to Bca cells specifically through the strong binding between the (D/N) GR motif and $\alpha \nu \beta 3$ integrin [31]. When using U87 (human glioma cells) and MDA-231 (human breast cancer cells), both of which expresses high level of $\alpha \nu \beta 3$ integrin [32], the amounts of PLZ4 internalized by U87 was higher than that in MDA231. The results indicates more significant expression difference of glycosylation or integrin between normal urinary tract cells and $\mathrm{BCa}$ cells [10]. Furthermore, five amino acids, X2 (D), X4 (R), X7 (F), X3, and X6 (G) are proven to be critical amino acids for tumor-specific binding.

The P4 peptide sequence (NVFTVSP) shares the similar sequence with the conserved region of FGFR3c, and is expected to block the interaction between FGF9 and FGFR3c. The abnormal expression of FGF9 high-affinity receptor FGFR3c is closely related to the tumorigenesis and progression of $\mathrm{BCa}$ [33-35]. Thus, the $\mathrm{P} 4$ peptide is potential for inhibiting the downstream pathway of FGFR3c.

\section{Other Targeting Mechanisms}

Among all CPPs, R11 is the best candidate for BCatargeting regardless of the cell variety [36]. But the mechanism remains to be explored. The high amount of phosphatidylserine and the overexpressed glycoprotein located on the outer surface of the $\mathrm{BCa}$ cell membrane might contributed to its targeting effiency.

The CLT1 peptide gains the ability to target the $\mathrm{BCa}$ in the presence of a urothelial tumor matrix component fibronectin, and then the cellular internalization can be driven through the $\alpha 5 \beta 1$ integrin and chloride intracellular channel 3 (CLIC3). For the purpose of CLT1 internalization, the origin of fibronectin appears to be irrelevant, as both plasma fibronectin, cellular fibronectin and urine fibronectin are able to work [37]. 
Table 2 The advantage and disadvantage of different targeting agents

\begin{tabular}{|c|c|c|}
\hline Name & Advantages & Disavantages \\
\hline $\begin{array}{l}\text { Nucleic acid } \\
\text { aptamers }\end{array}$ & $\begin{array}{l}\text { 1. Can be screened out to target many types of elements, including } \\
\text { inorganic and organic small molecules biological } \\
\text { macromolecules, and even cells } \\
\text { 2. Easy to produce and cheap }\end{array}$ & $\begin{array}{l}\text { 1. Challenging to obtain nucleic acid aptamers with high } \\
\text { specificity and high affinity for individual targets } \\
\text { 2. Binding affinity for electronegative target wanes } \\
\text { 3. Requires certain ionic strength, pH } \\
\text { 4. Easily degraded }\end{array}$ \\
\hline Antibodies & 1. Good performance in good specificity & $\begin{array}{l}\text { 1. limited tissue penetration } \\
\text { 2. high production cost }\end{array}$ \\
\hline Peptides & $\begin{array}{l}\text { 1. Smaller in size and penetrate more efficiently into tissue } \\
\text { compared to antibodies. } \\
\text { 2. Synthesized by automated techniques with low } \\
\text { production costs. }\end{array}$ & $\begin{array}{l}\text { 1. Have low stability in plasma, are sensitive to } \\
\text { proteases and can be cleared from the circulation in a } \\
\text { few minutes. }\end{array}$ \\
\hline
\end{tabular}

Neurotensin-quadruple neurotensin peptide (NT4) is a derivative of Neurotensin, and its branched structure protects it from the proteolysis and brings enhanced biological activity [38]. In vitro cell lines and tissue specimen proved the BCa-targeting property of NT4, but whether the neurotensin receptor is the real binding site remains unknown, as the neurotensin receptor coexist under the urothelial and in the smooth muscle layer.

\section{Application of Peptides}

\section{The Diagnostic Effect of Peptides on Bladder Tumors}

Combining the BCa-targeting peptide with fluorescein or imaging agents enable more precise visualization of $\mathrm{BCa}$ in different modalities.

pHLIP conjugated with a near-infrared fluorescent dye [indocyanine green (ICG)] targets low extracellular $\mathrm{pH}$, allowing visualization of malignant lesions in human bladder carcinoma ex vivo. Cystectomy specimens obtained after radical surgery were irrigated with a solution of the ICG pHLIP construct, and the fluorescence spot image was collected to establish the correlation between ICG pHLIP imaging and histopathological analysis. The detection sensitivity is $97 \%$. The specificity is $100 \%$, but reduced to $80 \%$ when transurethral resections or chemotherapy is applied prior to the CG pHLIP imaging [39].

$\mathrm{R} 11$, as an unique BCa-targeting $\mathrm{CPP}$, is used to functionalize the superparamagnetic iron oxide particles (SPIO) to improve the MR imaging contrast. Results from Prussian blue staining and TEM revealed that SPIO-R11 were efficiently taken up by BCa cells rather than normal urothelial cells, and in vitro MRI studies showed that SPIO-R11 greatly increased MR imaging contrast over the SPIO in $\mathrm{BCa}$ cells [25].

PLZ4 (amino acid sequence: cQDGRMGFc) was identified that could selectively bind to Bca cell lines and three primary Bca cells from human patients, but not to normal urothelial cells, cell mixtures from normal bladder specimens, fibroblasts, and blood cells [16]. After verifying the non-targeting properties of FITC, it can be connected to PLZ4 to detect cancer cells that shed urine.

\section{Application in Treatment}

The CLT1 peptide selected from the phage peptide library is a tumor homing peptide related to the clotted plasma of tumor stroma [40]. An in-depth understanding of peptides found that it can induce unfolded protein response and autophagy cell death in proliferating endothelial cells, thereby possessing antiangiogenic activity in vivo [41]. Also, the cytotoxicity of fibronectin and CLT1 peptide is significantly increased after forming a complex. The P4 peptide, with its antitumor properties, also screened through the phage peptide library, binds to FGF9 to counteract the aggressive phenotype induced by FGF9, including cell migration, invasion, and proliferation in vitro and inhibition of tumor growth in vivo. In research, FGF9 reduces the function of cisplatin-induced apoptosis to enhance drug resistance, and the antagonism of P4 to FGF9 can effectively enhance drug sensitivity [42].

Previous studies have shown that the peptide at the C-terminus of p53 (p53C) can restore the binding and transactivation functions of the mutated p53-specific DNA sequence and cause p53-related tumor cell apoptosis [43]. p53C itself cannot enter tumor cells spontaneously to play a role. Incorporating p53C specifically into tumor cells is the direction of our research. Nonpolar and large macromolecules cannot pass through the cell membrane, but R11 can transport hydrophilic substances and macromolecular proteins. $\mathrm{R} 11$ binds to $\mathrm{p} 53 \mathrm{C}$ to deliver $\mathrm{p} 53 \mathrm{C}$ to $\mathrm{Bca}$ cells. The results fully prove that $\mathrm{R} 11$ (R11-p53C) binding peptide can effectively target in situ and metastatic bladder tumor cells and inhibit tumor cell growth [42]. The $\mathrm{R} 11-\mathrm{p} 53 \mathrm{C}$ peptide is a potential drug for bladder urothelial 
cancer treatment, whether for in situ or metastatic bladder tumors.

Liposome polyethylene glycol can enhance the stability of particles in the bladder cavity and prevent particle aggregation, but how to solve low retention in the treatment of bladder tumors remains to be explored. Teams have developed targeted preparations, such as micellar nanocarriers surface-modified PLZ4 ligand and liposome-linked RWFV peptides to form peptide biopolymers. The advantage of these carriers is that they can encapsulate different therapeutic drugs and imaging agents. And it can be targeted to bladder tumor tissue, which can be used for tumor imaging and flexible replacement of drugs [43].

In addition to coupling with nanocarriers that encapsulate tumor chemotherapy drugs, peptides can also be directly coupled to antitumor drugs. The three lysine cores of NT4 provide other linking groups that can be used to connect other functional units [39]. Under the endoscope, the NT4 peptide is combined with a fluorescent group first, and the tumor is labeled and then combined with the chemotherapy drug. A simple "exchange" of functional units can achieve individualized treatment. Whether it is through nanocarriers or directly coupled with antitumor drugs, the tumor localization function of targeting peptides is used to internalize antitumor drugs into target tumor tissues, which not only improves the level of intracellular medications but also reduces systemic side effects.

\section{Conclusions and Future Perspectives}

The targeted polypeptide is an amino acid sequence that binds explicitly to different targets. It has become a hot research topic in recent decades and has a wide range of applications in biomedicine. Compared with the existing urine exfoliated cell line examination, the targeting peptide for Bca has higher sensitivity. The combined use with cystoscope significantly improves the tumor detection rate of the cystoscope. Targeting peptides have good target tissue positioning and internalization capabilities. After being mixed with imaging agents, it can effectively detect tumors that are not easy to find in the early stage, and after replacing the treatment ligand, it plays the role of targeted therapy, reducing the systemic side effects of the drug. Although targeted peptides have many advantages, they also face some problems. For example, how to maintain the stability of peptide molecules in the body and control the rate of degradation. Although polypeptides made from D-amino acids are more stable than other structure amino acids, they are not suitable for specific receptor binding applications due to the change in chirality. With the deepening of research, targeted peptides are expected to be used in the early diagnosis of Bca, targeted therapy, and follow-up examinations, and provide new methods for diagnosing and treating Bca.

In summary, most types of targeting peptides were screened out by involving specific types of cells as models, thus lead to Their targeting approaches noneffective in covering some aspects of tumor-targeting mechanisms, that is to say, these peptides might be that nonfunctional when exposed to tumor heterogeneity. Behaviors of targeting peptides and their complexes with cargos were different. The size and physiochemical properties of cargos might interfere with the targeting pathway. Current researches focus little on the role of targeting peptides in tumor promoter or inhibitor, exposing the unpredictable risk. Although the current targeting peptides still have shortcomings, their infinite potential deserves more attention. Once these targeted peptides are approved for clinical use, they will be of great help to the diagnosis, treatment, and follow-up examination of Bca.

\section{Compliance with Ethical Standards}

Conflict of interest The author(s) declared no potential conflicts of interest with respect to the research, authorship, and/or publication of this article.

Publisher's note Springer Nature remains neutral with regard to jurisdictional claims in published maps and institutional affiliations.

Open Access This article is licensed under a Creative Commons Attribution 4.0 International License, which permits use, sharing, adaptation, distribution and reproduction in any medium or format, as long as you give appropriate credit to the original author(s) and the source, provide a link to the Creative Commons license, and indicate if changes were made. The images or other third party material in this article are included in the article's Creative Commons license, unless indicated otherwise in a credit line to the material. If material is not included in the article's Creative Commons license and your intended use is not permitted by statutory regulation or exceeds the permitted use, you will need to obtain permission directly from the copyright holder. To view a copy of this license, visit http://creativecommons. org/licenses/by/4.0/.

\section{References}

1. Justin, M., Mark, S., \& Alfred, W. J., et al. (2020). Risk-adapted management of low-grade bladder tumours: recommendations from the International Bladder Cancer Group (IBCG). BJU International, 125, 497-505.

2. Kamat, A. M., Hegarty, P. K., \& Gee, J. R., et al. (2013). ICUDEAU International Consultation on Bladder Cancer 2012: screening, diagnosis, and molecular markers. European Urology, 63, 4-15.

3. Cai, S., Yan, J., \& Xiong, H., et al. (2018). Investigations on the interface of nucleic acid aptamers and binding targets. The Analyst, 143, 5317-5338.

4. Bayat, P., Nosrati, R., \& Alibolandi, M., et al. (2018). SELEX methods on the road to protein targeting with nucleic acid aptamers. Biochimie, 154, 132-155. 
5. Ryan, J. M., Wasser, J. S., \& Adler, A. J., et al. (2016). Enhancing the safety of antibody-based immunomodulatory cancer therapy without compromising therapeutic benefit: can we have our cake and eat it too? Expert Opinion on Biological Therapy, 16, 655-674.

6. Kobus, T., Vykhodtseva, N., \& Pilatou, M., et al. (2016). Safety validation of repeated blood-brain barrier disruption using focused ultrasound. Ultrasound in Medicine \& Biology, 42, 481-492.

7. Thiele, K., Ioannis, Z. K., \& Zhang, Y. Z., et al. (2016). Growth inhibition in a brain metastasis model by antibody delivery using focused ultrasound-mediated blood-brain barrier disruption. $J$ Control Release, 238, 281-288.

8. Smith, G. P. (1985). Filamentous fusion phage: novel expression vectors that display cloned antigens on the virion surface. Science (New York, N.Y.), 228, 1315-1317.

9. Scott, J. K., \& Smith, G. P. (1990). Searching for peptide ligands with an epitope library. Science (New York, N.Y.), 249, 386-390.

10. Wang, J., Tan, X., \& Guo, Q., et al. (2020). FGF9 inhibition by a novel binding peptide has efficacy in gastric and bladder cancer per se and reverses resistance to cisplatin. Pharmacological Research, 152, 104575.

11. Lee, S. M., Lee, E. J., \& Hong, H. Y., et al. (2007). Targeting bladder tumor cells in vivo and in the urine with a peptide identified by phage display. Molecular Cancer Research, 5, 11-19.

12. Knowles, L. M., Zewe, J., \& Malik, G., et al. (2013). CLT1 targets bladder cancer through integrin $\alpha 5 \beta 1$ and CLIC3. Molecular Cancer Research, 11, 194-203.

13. Yang, X., Zhang, F., \& Luo, J., et al. (2016). A new non-muscleinvasive bladder tumor-homing peptide identified by phage display in vivo. Oncology Reports, 36, 79-89.

14. Ks, L., Se, S., \& Em, H., et al. (1991). A new type of synthetic peptide library for identifying ligand-binding activity. Nature, $354,82-84$.

15. Lam, K. S., Lehman, A. L., \& Song, A., et al. (2003). Synthesis and screening of "one-bead one-compound" combinatorial peptide libraries. Methods in Molecular Biology, 369, 298-322.

16. Lin, T. Y., Li, Y. P., \& Liu, Q. Q., et al. (2016). Novel theranostic nanoporphyrins for photodynamic diagnosis and trimodal therapy for bladder cancer. Biomaterials, 104, 339-351.

17. Rao, B. D., Chakraborty, H., \& Chaudhuri, A., et al. (2020). Differential sensitivity of pHLIP to ester and ether lipids. Chemistry and Physics of Lipids, 226, 104849.

18. Jlenia, B., Chiara, F., \& Barbara, L., et al. (2015). Neurotensin branched peptide as a tumor-targeting agent for human bladder cancer. BioMed Research International, 2015, 1-7.

19. Pini, A., Falciani, C., \& Bracci, L. (2008). Branched peptides as therapeutics. Current Protein \& Peptide Science, 9, 468.

20. Khan, I. U., \& Beck-Sickinger, A. G. (2008). Targeted tumor diagnosis and therapy with peptide hormones as radiopharmaceuticals. Anticancer Agents in Medicinal Chemistry, 8, 186.

21. Lundquist, J. T., \& Dix, T. A. (1999). Preparation and receptor binding affinities of cyclic C-terminal neurotensin (8-13) and (913) analogues. Bioorganic \& Medicinal Chemistry Letters, 9, 2579-2582.

22. Young, L., Erin, K., \& Scott, C., et al. (2017). Targeting and internalization of liposomes by bladder tumor cells using a fibronectin attachment protein-derived peptide-lipopolymer conjugate. Bioconjugate Chemistry, 28, 1481-1490.

23. Pugliese, A., Vidotto, V., \& Beltramo, T., et al. (2005). A review of HIV-1 Tat protein biological effects. Cell Biochemistry \& Function, 4, 223-227.

24. Vives, E., Brodin, P., \& Lebleu, B. (1997). A truncated HIV-1 Tat protein basic domain rapidly translocates through the plasma membrane and accumulates in the cell nucleus. The Journal of Biological Chemistry, 272, 16010-16017.
25. Ding, C., Wu, K., \& Wang, W., et al. (2017). Synthesis of a cell penetrating peptide modified superparamagnetic iron oxide and MRI detection of bladder cancer. Oncotarget, 8, 4718-4729.

26. Janessa, W., Eden, S. L., \& Elizabeth, B., et al. (2020). pHDependent grafting of cancer cells with antigenic epitopes promotes selective antibody-mediated cytotoxicity. The Journal of Medicinal Chemistry, 63, 3713-3722.

27. Anderson, M., Moshnikova, A., \& Engelman, D. M., et al. (2016). Probe for the measurement of cell surface $\mathrm{pH}$ in vivo and ex vivo. Proceedings of the National Academy of Sciences of the United States of America, 113, 8177-8181.

28. Adochite, R. C., Moshnikova, A., \& Golijanin, J., et al. (2016). Comparative Study of tumor targeting and biodistribution of $\mathrm{pH}$ (Low) insertion peptides (pHLIP $\left({ }^{\circledR}\right)$ Peptides) conjugated with different fluorescent dyes. Molecular Imaging and Biology, 18, 686-696.

29. Chitrak, G., Yue, R., \& Blake, M. (2018). Cooperative nonbonded forces control membrane binding of the $\mathrm{pH}$-low insertion peptide pHLIP. The Biophysical Journal, 115, 2403-2412.

30. Jovana, G., Ali, A., \& Anna, M., et al. (2016). Targeted imaging of urothelium carcinoma in human bladders by an ICG pHLIP peptide ex vivo. Proceedings of the National Academy of Sciences of the United States of America, 113, 11829-11834.

31. Pablo, S., \& Eliana, A. K. (2020). Targeting tumors using peptides. Molecules, 25, 808.

32. Wang, S. T., Chen, K. J., \& Wu, T. H., et al. (2010). Photothermal effects of supramolecularly assembled gold nanoparticles for the targeted treatment of cancer cells. Angewandte Chemie International Edition in English, 49, 3777-3781.

33. Dai, S., Zhou, Z., \& Chen, Z. (2019). Fibroblast growth factor receptors (FGFRs): structures and small molecule inhibitors. Cells, 8, 614-628.

34. Yu, J., Xu, Q. G., \& Wang, Z. G., et al. (2018). Circular RNA cSMARCA5 inhibits growth and metastasis in hepatocellular carcinoma. The Journal of Hepatology, 68, 1214-1227.

35. Zhang, X., Ibrahimi, O. A., \& Olsen, S. K., et al. (2006). Receptor specificity of the fibroblast growth factor family. The complete mammalian FGF family. The Journal of Biological Chemistry, 281, 15694-15700.

36. Du, Y., Wang, L., \& Wang, W., et al. (2018). Novel application of cell penetrating R11 peptide for diagnosis of bladder cancer. The Journal of Biomedical Nanotechnology, 14, 161-167.

37. Pankov, R. (2002). Fibronectin at a glance. The Journal of Cell Science, 115, 3861-3863.

38. Kim, J. H., Bae, C., \& Kim, M. J., et al. (2020). A novel nucleolin-binding peptide for cancer. Theranostics, 10, 9153-9171.

39. Jan, P., Darren, B. M., \& Masanobu, K., et al. (2006). Peptides selected for binding to clotted plasma accumulate in tumor stroma and wounds. Proceedings of the National Academy of Sciences of the United States of America, 103, 2800-2804.

40. Lynn, K. M., Gunjan, M., \& Brian, H. L., et al. (2012). CLT1 targets angiogenic endothelium through CLIC1 and fibronectin. Angiogenesis, 15, 115-129.

41. Lima, I., Navalkar, A., \& Maji, S. K., et al. (2020). Biophysical characterization of $\mathrm{p} 53$ core domain aggregates. The Biochemical Journal, 477, 111-120.

42. Zhang, T. T., Wu, K. J., \& Ding, C., et al. (2015). Inhibiting bladder tumor growth with a cell penetrating R11 peptide derived from the p53 C-terminus. Oncotarget, 6, 37782-37791.

43. Wang, H., Ding, S., \& Zhang, Z., et al. (2019). Cationic micelle: a promising nanocarrier for gene delivery with high transfection efficiency. The Journal of Gene Medicine, 21, e3101.

44. Roxin, A., \& Zheng, G. (2012). Flexible or fixed: a comparative review of linear and cyclic cancer-targeting peptides. Future Medicinal Chemistry, 4, 1601-1618. 
45. Falciani, C., Brunetti, J., \& Lelli, B., et al. (2013). Cancer selectivity of tetrabranched neurotensin peptides is generated by simultaneous binding to sulfated glycosaminoglycans and protein receptors. Medicinal Chemistry, 56, 5009-5018.
46. Falciani, C., Fabbrini, M., \& Pini, A., et al. (2007). Synthesis and biological activity of stable branched neurotensin peptides for tumor targeting. Molecular Cancer Therapeutics, 6, 2441-2448. 